\title{
High energy properties of PKS 1830-211
}

\section{Shu Zhang*a, Yu-peng Chen ${ }^{a}$, Werner Collmar ${ }^{b}$, Luigi Foschini ${ }^{c}$, Ti-Pei Li $^{a, d}$, Diego F. Torres ${ }^{e}$, Jian-Min Wang ${ }^{a, f}$}

${ }^{a}$ Key Laboratory for Particle Astrophysics, Institute of High Energy Physics, 19B YuQuan Road, Beijing 100049, China

${ }^{b}$ Max-Planck-Institut für Extraterrestrische Physik, P.O. Box 1603, 85740 Garching, Germany

${ }^{c}$ INAF/IASF-Bologna, via Gobetti 101, 40129, Bologna, Italy

${ }^{d}$ Center for Astrophysics, Tsinghua University, Beijing 100084, China

eICREA \& Institut de Ciències de l'Espai (IEEC-CSIC), Campus UAB, Facultat de Ciències, Torre C5-parell, 2 a planta, 08193 Barcelona, Spain

${ }^{f}$ Theoretical Physics Center for Science Facilities (TPCSF), CAS

E-mail: Szhang@ihep.ac.cn

We report on an analysis of X- and $\gamma$-ray observations of PKS $1830-211$, a gravitationallensed high-redshift quasar, based on the long-term campaigns carried out by INTEGRAL and COMPTEL. The INTEGRAL data currently available present a $33 \sigma$ significance detection in the $20-100 \mathrm{keV}$ band, while the COMPTEL 6-years data provide a $5.2 \sigma$ significance detection in the $1-3 \mathrm{MeV}$ energy band. At hard X-rays, INTEGRAL and supplementary SWIFT observations show flux variability on timescales of months. At $\gamma$-rays, the source shows persistent emission over years. The hard X-ray spectrum is well represented by a power-law model, with $\Gamma \sim 1.3$ in the $20-250 \mathrm{keV}$ band. This photon index is well consistent with the previous report of $\Gamma \sim 1.3$ obtained at $E>3.5 \mathrm{keV}$ from the best fit of XMM-Newton data with a broken power law model. The joint XMM-Newton/INTEGRAL spectrum presented here is fitted with a broken power-law model and the parameters are refined compared to the previous. The results show that the photon index changes from $\sim 1.0$ to $\sim 1.3$ at a break energy of $\sim 4 \mathrm{keV}$. At MeV energies, the spectrum softens to $\Gamma \sim 2.2$. These results, together with the EGRET measurement at $E \geq 100 \mathrm{MeV}$, constitute a broad-band spectrum containing the peak of the power output at $\mathrm{MeV}$ energies, similar to most high-luminosity $\gamma$-ray blazars. The measured spectral characterstics are then discussed in the framework of the gravitational lens effects.

\section{7th INTEGRAL Workshop}

September 8-11 2008

Copenhagen, Denmark

\footnotetext{
* Speaker.
} 


\section{Introduction}

Since the first gravitational lens candidate was detected in 1979, the total number of such system has grown. Among them is the high redshift blazar PKS 1830-211 $(z=2.507)$, gravitationally lensed by an intervening galaxy at $z=0.89$. The discovery of such gravitational system traces back to radio observations [1]. The radio map was composed of two compact components separated by $1^{\prime \prime}$, supposed to be the split images from the central region of the source and an extended structure which is most probably from the jet and regarded as an unusually strong Einstein ring [2]. Steppe et al. [3] have shown that PKS 1830-211 is radio variable on timescales of months.

The previous X-ray observations (Chandra and INTEGRAL) revealed a quite hard spectrum, modeled with a power-law with a photon index $1.09 \pm 0.05$ over the energy band $0.5-80 \mathrm{keV}$ [4]. $X M M$-Newton observations gave the best fit of a broken power-law model, with the photon index changing from $\sim 1.0$ to $\sim 1.3$ at energies around $3.5 \mathrm{keV}$ [5].

PKS $1830-211$ was included in the first INTEGRAL AGN catalogue by [6], with a photon index $1.96_{-0.24}^{+0.27}$ in the $20-100 \mathrm{keV}$ energy band and in the INTEGRAL extragalactic survey by [6], with a $20-100 \mathrm{keV}$ flux $\sim 3$ mCrab level averaged over the first 2.5 years of the INTEGRAL observations.

At MeV energies PKS $1830-211$ was firstly reported by [7]. The COMPTEL first 4-year observations (1991-1995) revealed a detection of $4.5 \sigma$ in the $1-3 \mathrm{MeV}$ band. Contemporaneously, PKS $1830-211$ was detected by EGRET at $\geq 100 \mathrm{MeV}$ with $7.8 \sigma$ significance and a photon index of $2.59 \pm 0.13$ [8].

Since the amount of INTEGRAL public data has been significantly increased after the last report, we carried out a detailed analysis of PKS 1830 - 211 with all available INTEGRAL data. We also reanalyzed the XMM-Newton data with a newer software version, which allows us to extend the analysis down to $0.2 \mathrm{keV}$. The data from COMPTEL and EGRET are also reanalyzed and added in order to obtain the best broad-band high-energy spectrum available to date.

\section{Observations and data analysis}

\subsection{INTEGRAL}

The available INTEGRAL observations comprise about 1095 science windows (scw), for a total exposure of $2500 \mathrm{ks}$. The analysis were performed by using the INTEGRAL OSA 7.0. All the sources within the FOV which are brighter or comparable to PKS 1830-211 were taken into account in extracting the source spectrum and light curve. An additional 3\% systematic error was added to the spectra because of calibration uncertainties. The spectra were fitted with XSPEC V 12.3. 1 and the model parameters were estimated at $90 \%$ confidence level.

The sum of all observations provide a detection with IBIS/ISGRI at $\sim 33 \sigma$ level in the $20-100 \mathrm{keV}$ energy band (Fig.1). The average $20-100 \mathrm{keV}$ flux is $0.75 \pm 0.03$ counts/s, corresponding to $\sim 3 \mathrm{mCrab}$. The spectrum of the entire ISGRI data set is well fitted $\left(\tilde{\chi}^{2}=0.59\right.$ with 8 degrees of freedom) by a power-law model with $\Gamma=1.29_{-0.15}^{+0.16}$ in the $20-250 \mathrm{keV}$ energy band. 


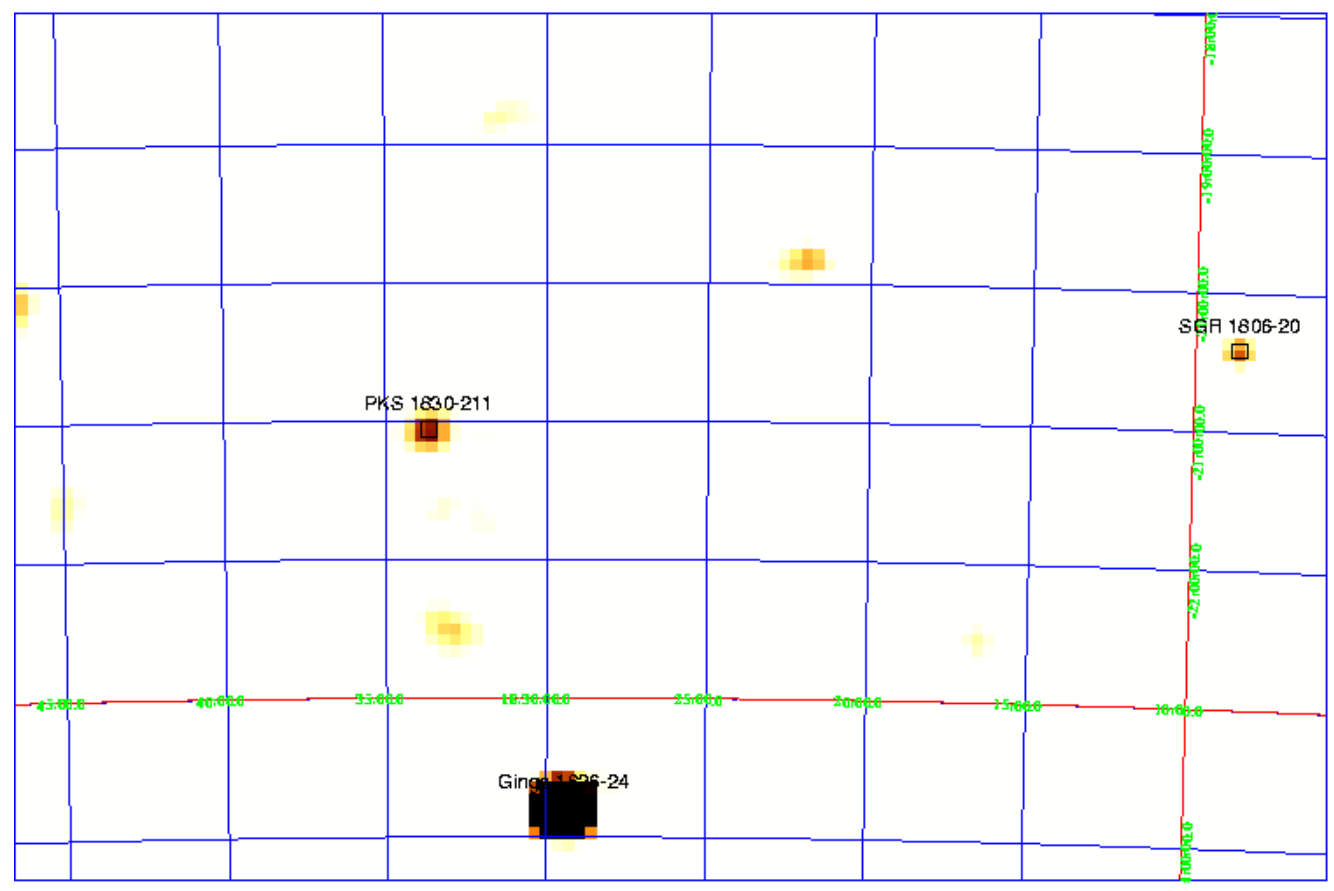

Figure 1: The ISGRI significance map of PKS $1830-211$ region in the $20-100 \mathrm{keV}$ band, obtained by combining the observations of $2003-2006$.

\subsection{XMM-Newton}

The available three XMM-Newton observations $(0204580201,0204580301,0204580401)$ are combined to obtain an average spectrum for the joint fit with ISGRI data. Although, the broken power-law and the log-parabola models can be fit with similar results in the $\chi^{2}$ test, the broken power-law is slightly better and we consider this model as the best fit.

\subsection{SWIFT}

Swift/BAT has a very large FOV and makes it possible for a source to be daily monitored at the hard X-rays. The data products are therefore the source lightcurves and are publicly available ${ }^{1}$.

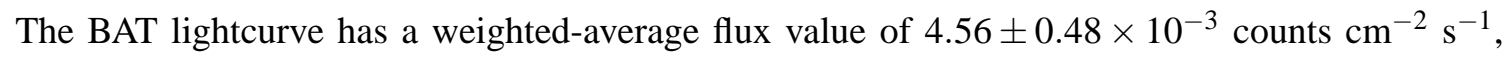
corresponding to $2 \mathrm{mCrab}$ at $\sim 9.5 \sigma$ over a time period of roughly 2.5 years.

\subsection{COMPTEL}

Here we take the complete 6-years COMPTEL data, until the second reboost of the satellite in 1997, after which the background changed a lot making it difficult for further research, to investigate again the $\mathrm{MeV}$ emission. These data are subdivided into the so-called $C G R O$ phases, with each period covering typically one year of observations. The source is again detected mainly in the $1-3 \mathrm{MeV}$ band, but the detection significance is improved to $5.2 \sigma$ (Fig.2).

\footnotetext{
${ }^{1}$ See the Swift/BAT transient monitor results provided by the SWIFT Team at http://swift.gsfc.nasa.gov/docs/swift/results/transients/
} 


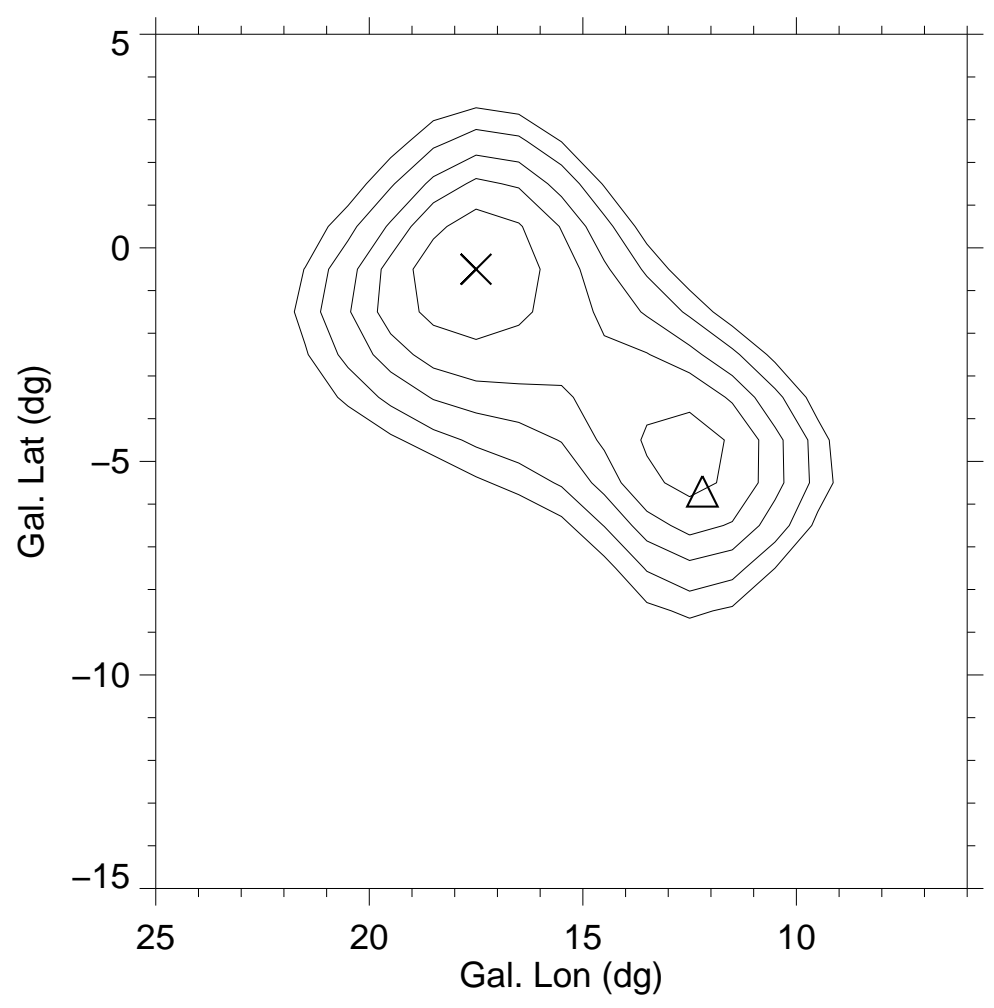

Figure 2: The 1-3 MeV map from COMPTEL observations of phases 1-6. The contours start at detection significance level of $3 \sigma$, with steps of $1 \sigma$. The triangle represents the location of PKS $1830-211$, and the cross the location of the so-called $\mathrm{MeV}$ source $l=18$, namely GRO J1823-12.

\section{Time variability}

The ISGRI lightcurve shows that the flux tends to drop smoothly during the first 2.5 years since 2003 and then to raise in the following years. A search for the flux variability on shorter time scales (4 days bins) resulted in two interesting episodes (around MJD 53050 and 53600). SWIFT/BAT data are combined in 10-days bins and the resulted lightcurve shows several time periods with persistent flux excess. Accordingly, the observations are divided again into these 6 long term intervals. The resulted lightcurve suggests that PKS $1830-211$ is rather variable at the hard X-rays on the time scale of months. The lightcurves from COMPTEL $(1-3 \mathrm{MeV}$, time period 1991 - 1997) and EGRET ( $\geq 100 \mathrm{MeV}$, time period 1991 - 1995), with each bin presenting the average of one CGRO satellite observing phase, indicate that PKS $1830-211$ is likely to have persistent emission over years at $\gamma$-rays. The details of the lightcurves refer to [9]. 


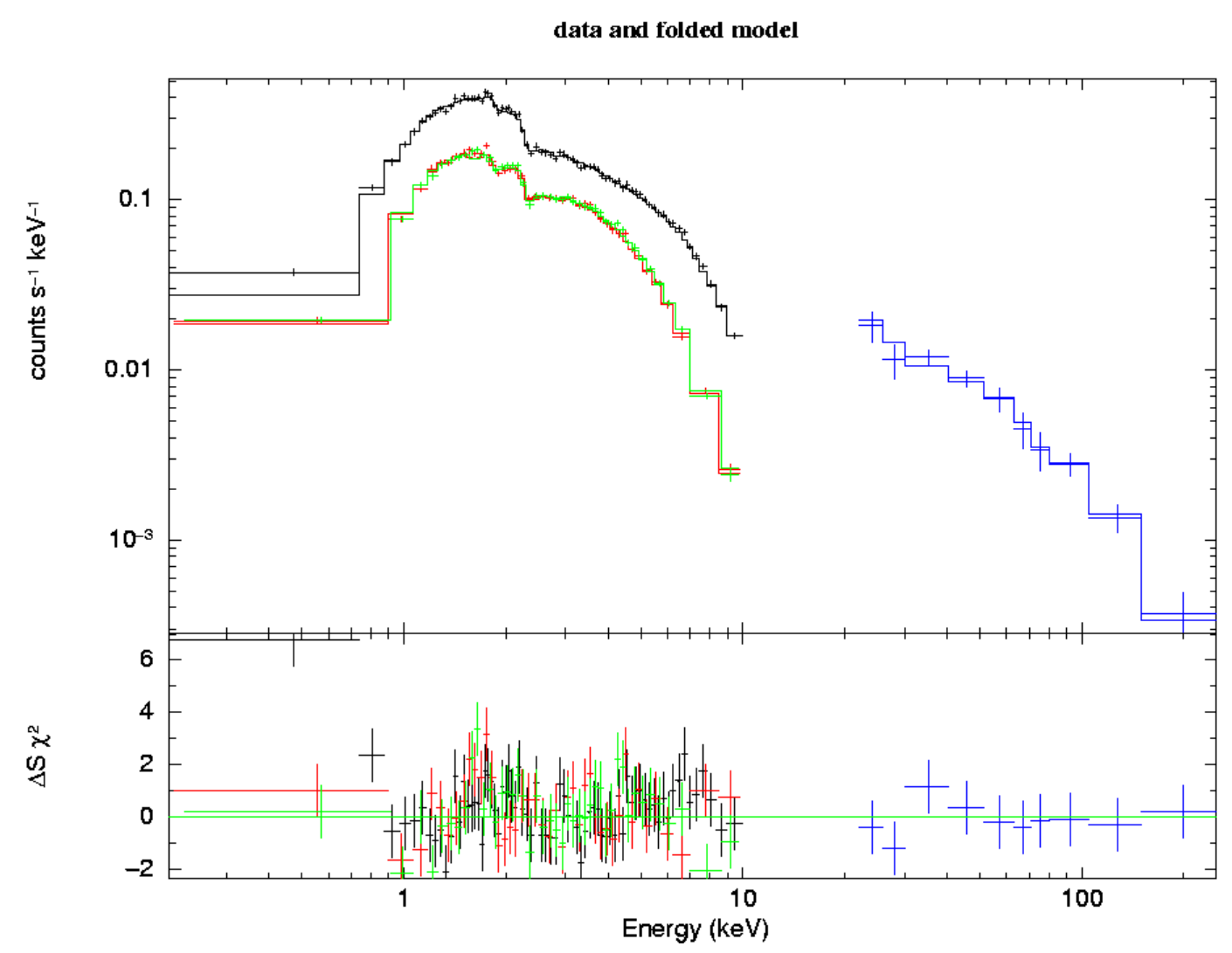

Figure 3: Spectral fit with a broken power law model for the combined XMM-Newton data (at energy below $10 \mathrm{keV}$, with the black for PN and the red/green for MOS), and the ISGRI data (at energy above $20 \mathrm{keV}$ ). For better visibility the XMM-Newton spectra have been rebinned in the plot.

\section{Broad-band energy spectrum}

We performed a joint fit of the XMM-Newton/INTEGRAL data. The source luminosity in the $0.2-250 \mathrm{keV}$ energy band is of $3.5 \times 10^{48} \mathrm{erg} \mathrm{s}^{-1}$. The broken power-law model results to be again the best fit model, with an improvement with respect to the single power-law model $>99.99 \%$ as calculated with the $F$-test. The results show the photon index changes from $\sim 1.0$ to $\sim 1.3$ at a break energy $\sim 4 \mathrm{keV}$ (Fig.3). We notice that, although the overall spectrum is well fitted with a reduced $\chi^{2}$ derived as $\sim 1.04$, there may exists an evidence for Iron line red-shifted from $6.4 \mathrm{keV}$ to $1.8 \mathrm{keV}(\mathrm{Z}+1=3.5)$. At $\mathrm{MeV}$ energies the spectrum can be well represented ( $\tilde{\chi}^{2}=0.4$ for 2 dof) by a single power-law model with $\Gamma=2.23_{-0.27}^{+0.36}$ measured from COMPTEL data combined from $C G R O$ phases 1-4. Fig.4 shows the high-energy broad-band spectrum for the results derived in this paper, including the data from XMM-Newton and INTEGRAL to cover the soft/hard Xrays $(0.2-250 \mathrm{keV})$; from COMPTEL for the $\gamma$-rays $(0.75-30 \mathrm{MeV})$; and from EGRET for the $\gamma$-rays in the $\geq 100 \mathrm{MeV}$ energy band [8]. In such a broad-band view, the power output of PKS 1830 - 211 shows a bump located at MeV energies, as expected in the common view of a high-luminosity blazar, where the high-energy part of the spectral energy distribution (SED) is due to inverse-Compton emission from the relativistic electrons in a jet scattering off seed photons coming from a source external to the jet. 


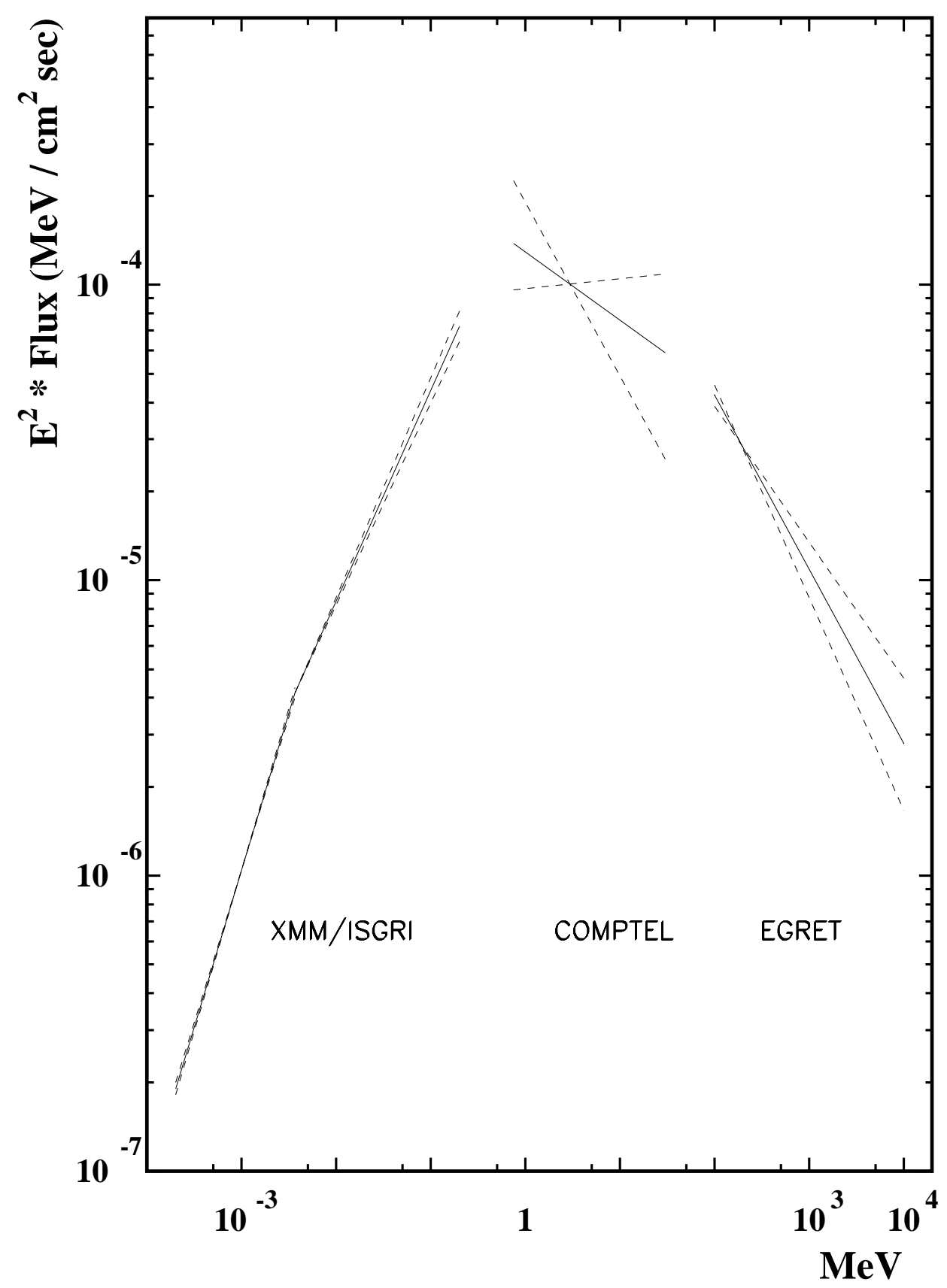

Figure 4: The broad energy spectrum of PKS $1830-211$. The solid lines are the broken power law shape as obtained by XMM-Newton/INTEGRAL at X-rays, power law shapes by COMPTEL at MeV energies, and by EGRET at $\geq 100 \mathrm{MeV}$ [8]. The dashed lines represent the $1 \sigma$ error in spectral index. 


\section{Discussion and summary}

The most interesting feature in the broad-band high-energy spectrum of PKS $1830-211$ is the spectral flattening below $\sim 4 \mathrm{keV}$. Such a flattening has been observed also by [4] in the combined Chandra/INTEGRAL spectrum, but the best fit model proposed is a single power-law with $\Gamma=$ $1.09 \pm 0.05$ extending over the entire $0.5-80 \mathrm{keV}$ band absorbed by cold gas from the intervening galaxy at $z=0.89$, with column density $N_{\mathrm{H}}^{z} \sim 2 \times 10^{22} \mathrm{~cm}^{-2}$. Instead, in the present work, by analyzing a spectrum covering a wider energy range $(0.2-250 \mathrm{keV})$, we have shown evidence of a photon deficit at low energies in addition to the absorption from the intervening galaxy, confirming and extending the results obtained with XMM-Newton only $(0.4-10 \mathrm{keV})$ reported by [5]. This low-energy photon deficit can be best fit with a power-law harder $(\Gamma \sim 1.0)$ than the one at energies greater than $\sim 4 \mathrm{keV}(\Gamma \sim 1.3)$.

However, in the case of PKS 1830 - 211, the gravitational lensing should have an impact on the spectral and variability properties of the source, but it is not clear how to weight it at highenergy. These effects in the $\gamma$-ray band on distant blazars have been discussed by [10] and [11]. The observed hard X-rays and, probably the soft X-rays as well, are the combination of the contributions from SSC and EC, which in turn are generated from different places. Therefore, the lensing can act differently, resulting in changes in spectral shape.

In summary, we presented here the most up-to-date broad-band high-energy spectrum of PKS $1830-211$. The source presents a low-energy roll-off that can be explained efficiently in terms of a natural interplay between SSC and EC, as shown in other high-z Flat Spectrum Radio Quasars (FSRQ) [12]. However, it is not clear what is the weight of the amplification factor due to the gravitational lensing. Future observations at $\mathrm{X}$-rays with higher spatial resolution should allow us to measure this factor. More details can be found in [9].

\section{References}

[1] Pramesh Rao A. \& Subrahmanyan R., 1830-211 - A flat-spectrum radio source with double structure, MNRAS 231229 (1988)

[2] Jauncey, D. L., Reynolds, J. E., Tzioumis, A. K., et al., An unusually strong Einstein ring in the radio source PKS1830 - 211, Nature 352132 (1991)

[3] Steppe, H., Paubert, G., \& Sievers, A., Millimeter continuum measurements of extragalactic radio sources - part three, A\&AS 102611 (1993)

[4] De Rosa, A., Piro, L., Tramacere, A., et al., The broad-band X-ray spectrum of the blazar PKS B1830-211 by Chandra and INTEGRAL, A\&A 438121 (2005)

[5] Foschini, L., Ghisellini, G., Raiteri, C.M., et al., XMM-Newton observations of a sample of gamma-ray loud active galactic nuclei, A\&A 453829 (2006)

[6] Beckmann, V., Gehrels, N., Shrader, C., R., et al., The First INTEGRAL AGN catalog, ApJ 638642 (2006)

[7] Collmar, W., New blazar detections at MeV energies by COMPTEL, ASPC 350120 (2006)

[8] Hartman, R. C., Bertsch, D. L., Bloom, S. D., et al., The third EGRET catalog of high-energy gamma-ray sources, ApJS 12379 (1999) 
[9] Zhang, S., Chen Yu-Peng, Werner Collmar, et al., High energy properties of PKS 1830-211, ApJ 685 400 (2008)

[10] Combi J.A. \& Romero G. E. 1998, A\&AS 128, 423 Observations of the radio emission field around the gamma -ray source 2EG J1834-2138, A\&AS 128423 (1998)

[11] Torres, D. F., Romero, G. E. \& Eiroa, E., Gravitational lensing as a possible explanation for some unidentified gamma-ray sources at high latitudes, ApJ 569600 (2002)

[12] Worsley, M.A., Fabian, A.C., Turner, A.K., et al., XMM-Newton confirmation of a warm absorber in the $z=4.4$ blazar PMN J0525-3343, MNRAS 350207 (2004) 